\title{
COLONIZACIÓN Y SUCESIÓN DE NUEVOS MEDIOS ACUÁTICOS I. COMPOSICIÓN Y ESTRUCTURA DE LAS COMUNIDADES DE INSECTOS
}

\author{
J. Velasco, A. Millán y L. Ramírez-Díaz \\ Departamento de Biología Animal y Ecología, Facultad de Biología, Universidad de Murcia. Campus Universitario de Espinardo, \\ 30100 Murcia, España.
}

Palabras clave: Colonización, sucesión primaria, insectos acuáticos, comunidad, estructura.

\author{
ABSTRACT \\ COLONIZATIONAND SUCCESSION OF NEW AQUATIC SYSTEMS \\ I. COMPOSITION AND STRUCTURE OF INSECT COMMUNITIES
}

\begin{abstract}
A study of colonization and primary succession of aquatic insect communities was carried out during an annual cycle in a set of ten small new ponds. Ponds were of similar size but differed initially in some environmental conditions: water regime, filling time, substrate composition, presence and type of aquatic vegetation, insolation, nutrient and dissolved salt concentration. This paper, the first of two presented, analyzes the general composition and structure of insect communities in the different ponds, in terms of composition, richness, frequency, relative abundance, diversity and evenness. Insect communities of ponds presented a similar composition and structure, except in ponds subjected to temporary regime (pond VIII, $1^{\text {st }}$ period), or with saline water (pond X). The stress effect of theses environmental factors reduced significantly the number of species colonizing. Chironomids were the most important group in number of species and individuals. Cloeon dipterum (EPHEMEROPTERA), Culiseta longeareolata and Procladius sagittalis (DIPTERA) were the fundamental components of communities. They colonized and reproduced several generations in the freshwater ponds.
\end{abstract}

\section{INTRODUCCIÓN}

Generalmente, sucesión y colonización se han considerado procesos equivalentes y difícilmente separables, aunque el termino sucesión incluye la colonización y los cambios subsecuentes (FISHER, 1983). SHELDON (1984) define la colonización como la secuencia de sucesos que conducen al establecimiento de individuos, poblaciones, especies o grandes grupos, en lugares donde estaban ausentes, al menos temporalmente. El termino sucesión se refiere a los cambios que se producen en la composición y estructura de las comunidades después de una discreta y, normalmente, impredecible perturbación en un medio determinado (FISHER, 1990). Si esta perturbación es tan intensa que elimina por completo la comunidad establecida, se produce un proceso de sucesión primaria, aunque normalmente en medios naturales tienen lugar procesos de sucesión secundaria. En cambio, los sistemas acuáticos artificiales constituyen excelentes medios para el estudio de los procesos de colonización y sucesión primaria, ya que carecen de una historia previa y a la vez pueden ser manipulados fácilmente en función de los objetivos planteados por el investigador.

La sucesión primaria, entendida como los procesos de colonización y los subsecuentes cambios en la estructura y organización de las comunidades en medios nuevos que no han sufrido anteriormente la influencia de una comunidad (BEGON et al.,1988), ha sido muy poco estudiada en medios acuáticos aislados de pequeño tamaño. Destacan los estudios realizados con Quironómidos, en charcas formadas en hoyos procedentes de extracción de grava (STREET \& TITMUS, 1979), y con macroinvertebrados y macrófitos, en charcas de extracción de arcilla (BARNES, 1983; FRIDAY, 1987).

La colonización aérea, tanto por la entrada activa de adultos, como por ovoposición, es una de las principales vías de poblamiento de ríos (WILLIAMS \& HYNES, 1976a; WILLIAMS, 1980; BIRD \& HYNES, 1981a; MULLER, 1982; MILNER, 1987; MALMQVIST, 1991), medios acuáticos temporales (WILLIAMS, 1977, WIGGINS et al.; 1980, McLACHLAN \& 
CANTRELL, 1980; GRAY \& FISHER, 1981; FISHER et al., 1982; WILLIAMS, 1985; WILLIAMS, 1987), embalses (PATERSON \& FERNANDO, 1969a) y la única que opera en cuerpos de agua aislados de nueva creación (BARNES, 1983), como los estanques objeto de este estudio. La mayoría de insectos acuáticos, en estado adulto, son capaces de volar y dispersarse a otros medios, siendo este comportamiento una parte de su ciclo de vida y una adaptación para la supervivencia, relacionada fundamentalmente, con la reproducción y la alimentación (JOHNSON, 1969).

Durante un ciclo anual, se estudiaron los procesos de colonización y sucesión primaria en una serie de estanques con diferentes condiciones ambientales. En este primer articulo, se analiza la composición y estructura general de las comunidades de insectos de los diferentes estanques. En un segundo articulo (en el mismo volumen), se analizan los cambios temporales, estableciéndose la secuencia de colonización y determinando las diferencias más importantes en relación a las características ambientales consideradas.

\section{MATERIAL Y MÉTODOS}

Durante un ciclo anual (10-XI-198317-XII-1984) se estudiaron los procesos de colonización y sucesión de las comunidades de insectos acuáticos en un conjunto de 10 estanques de igual tamaño pero diferentes respecto al grado de insolación, sustrato, presencia y tipo de vegetación, concentración de nutrientes, salinidad, régimen hídrico y época de llenado.

Los estanques, ubicados en el Campus Universitario de Espinardo (Murcia), son pequeñas cubetas rectangulares de hormigón, de dimensiones $178.5 \mathrm{~cm}$ x $63.5 \mathrm{~cm}$ x $34.5 \mathrm{~cm}$ y con una capacidad de 390 1. Están situados al aire libre y dispuestos, uno a continuación del otro, en línea recta, orientados en dirección SO-NE. Dichos estanques fueron enumerados en orden creciente del I al X. La situación y caracterización inicial de cada estanque, se representa en la Fig. 1.

Con respecto al número de horas de sol que reciben, hay una gradación de menor a mayor, desde el estanque I al X,

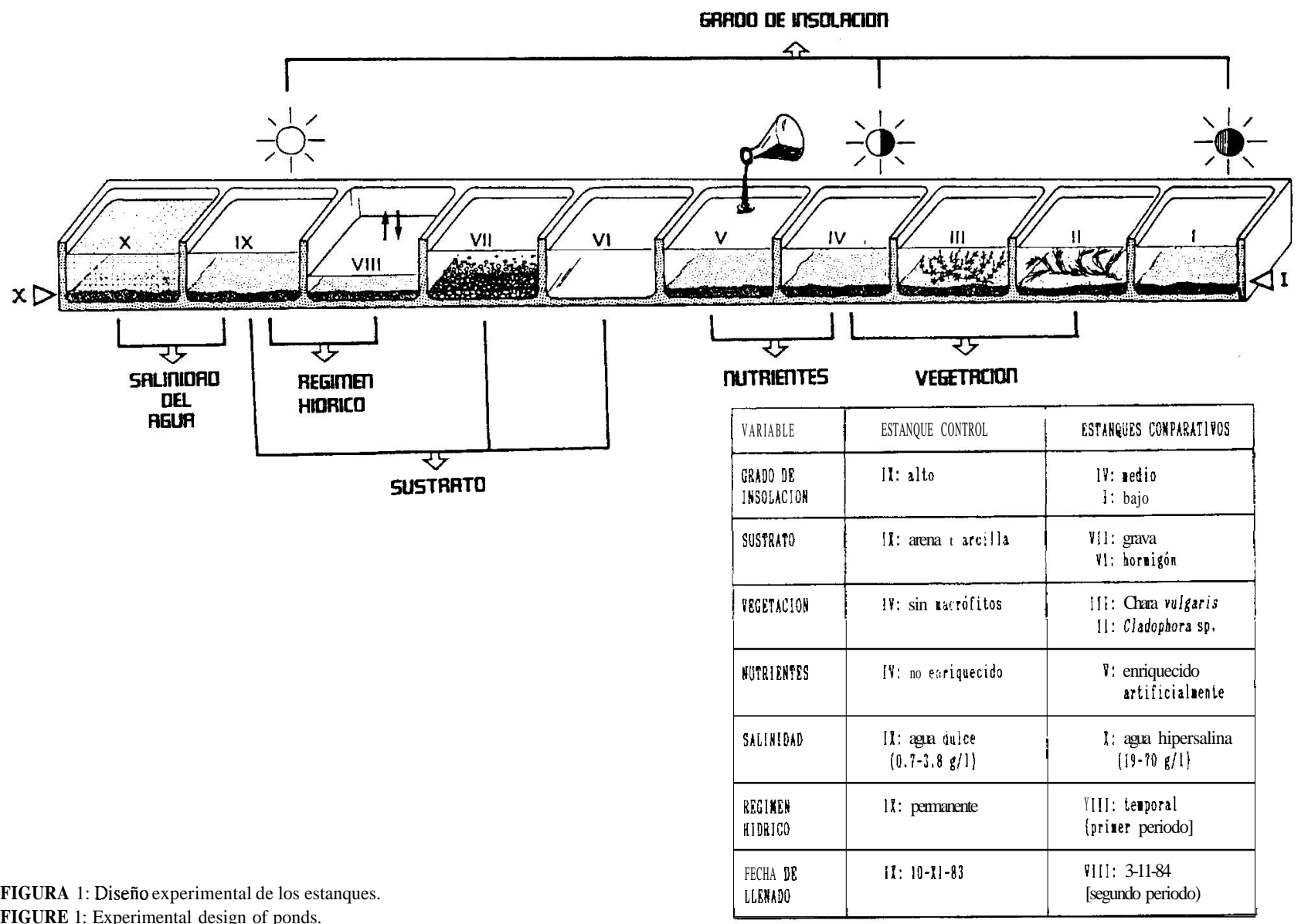


determinada por la presencia de una pared vertical de $3 \mathrm{~m}$ de altura, al lado del primer estanque, que le da sombra y le resta visibilidad.

A todos los estanques, excepto al VI y VII, se les añadió una capa uniforme de $2 \mathrm{~mm}$ de sedimento, constituido por una mezcla de arena fina y arcilla a igual proporción, antes de que se llenaran con agua. En cambio, al estanque VII se le incorporó una capa de grava de $1 \mathrm{~cm}$ de altura, mientras que en el estanque VI quedó el hormigón, como único sustrato. Además se adicionó a cada uno de ellos 50 gramos de tierra de jardín, rica en materia orgánica, con el fin de estimular la llegada de las primeras especies colonizadoras.

Los estanques se llenaron hasta su nivel máximo $(31 \mathrm{~cm}$ de profundidad) el 10 de Noviembre de 1983. Los nueve primeros con agua potable de suministro público, previamente depurada con un filtro de carbón activo para eliminar el cloro presente. El estanque $\mathrm{X}$, en cambio, se llenó con dos tercios de agua hipersalina (75 g/l), procedente de una rambla salada próxima (Rambla de Fortuna, Murcia), previamente filtrada con un colador de $250 \mu \mathrm{m}$ de luz de malla, y un tercio de agua potable depurada.

Todos los estanques, excepto el VIII, se mantuvieron en un régimen permanente de agua durante el periodo de estudio, rellenándolos con agua potable hasta el nivel máximo fijado, tras cada muestreo. El estanque VIII, por el contrario, después de llenarse inicialmente, no se volvió a rellenar de agua, dejando que se secara completamente. Posteriormente, en una segunda fase, se añadió agua nuevamente, el 3 de Agosto de 1984, sometiéndolo al mismo régimen de llenado que el resto de estanques hasta el final del periodo de estudio.

Tras su llenado, al estanque II se le añadieron filamentos de Cladophora sp. y al III, varias plántulas de Chara vulgaris. Estos macrófitos, procedentes de medios acuáticos cercanos, se lavaron e inspeccionaron bajo la lupa binocular, antes de ser introducidos, eliminando las puestas, larvas y adultos de organismos acuáticos adosados a ellos. El estanque V, se enriqueció artificialmente de nutrientes, mediante la adición de $500 \mathrm{ml}$ de medio WOY, $2500 \mathrm{ml}$ de solución patrón de boro y $200 \mathrm{ml}$ TRIS para tamponar el medio (ROS, 1979).

A lo largo del estudio, la toma de muestras se realizó con una periodicidad aproximadamente quincenal en otoño e invierno, y semanal en los meses de primavera y verano, realizándose un total de 34 muestreos. Para el muestreo de la taxocenosis de insectos acuáticos, dadas las características de estos medios (hábitats homogéneos de pequeñas dimensiones y escaso sedimento), se diseñó una unidad de muestreo (modificación del muestreador Gerking), de metacrilato transparente sellado con silicona y sección cuadrangular de $35 \mathrm{~cm}$ de lado y
$40 \mathrm{~cm}$ de altura, siendo su área la doceava parte de la superficie total de un estanque. El tamaño de la muestra consistió en una unidad de muestreo para el bentos y en dos unidades para el necton. Las exuvias y adultos aéreos emergidos o caídos, presentes en la superficie del agua fueron recogidos con una pequeña manga entomológica.

Además, se midieron un total de 28 parámetros ambientales, de los cuales, dos son climáticos ( $T^{\circledR}$ máxima y mínima del aire) y 26 hacen referencia a la composición fisico-química y biológica del agua de cada estanque: $T^{\text {a }}$ puntual, máxima y mínima del agua, volumen de agua, volumen de sedimento, sólidos en suspensión, transparencia, evaporación, pH, conductividad, salinidad, alcalinidad, oxígeno disuelto, saturación de oxígeno, nitratos, nitritos, amonio, fosfatos, silicatos, sulfatos, cloruros, dureza total, calcio, magnesio, clorofila "a" y D430/D665. Los valores de dichos parámetros a lo largo del periodo de estudio pueden ser consultados en VELASCO (1989).

Los datos faunísticos obtenidos fueron expresados como número total de individuos, de cada una de las especies presentes en cada estanque, así como de sus diferentes estados de desarrollo y exuvias ninfales.

Para la descripción de la composición y estructura de las comunidades de insectos acuáticos de cada estanque se han utilizado los siguientes índices ecológicos: Riqueza de especies (S), Frecuencia relativa (F\%), Dominancia o abundancia relativa $(\mathrm{D} \%)$, Diversidad $\left(\mathrm{H}^{\prime}\right)$ y Equitabilidad (E). El índice de diversidad empleado ha sido el de Shannon-Wiener y la equitabilidad fue calculada usando la fórmula de PIELOU (1977).

\section{RESULTADOS}

\section{El medio abiótico}

El clima en la zona de estudio, se caracteriza por escasas e irregulares precipitaciones $(\approx 300 \mathrm{~mm}$ anuales) y un elevado régimen de temperaturas $\left(18.27{ }^{\circ} \mathrm{C}\right.$ de media anual $)$, propio de las áreas más áridas de la Península Ibérica. Aunque la pluviosidad presenta una alta variabilidad anual, los periodos húmedos por excelencia son primavera y otoño, donde se registran las máximas precipitaciones. El resto del año, las lluvias son muy escasas e irregulares.

El ambiente fisico-químico donde se van a establecer las especies está determinado, fundamentalmente, por la calidad y cantidad del agua de entrada, y las variaciones climáticas estacionales. Los nueve primeros estanques, llenados con agua potable de suministro público, presentan una composición fisico-química muy similar, caracterizada por su basicidad, una importante reserva alcalina, un cierto grado de mineralización 
y escasos nutrientes, salvo el estanque $\mathrm{V}$, al comienzo del estudio (VELASCO, 1986).

En la Fig. 2 aparece la ordenación de las muestras en el espacio definido por los tres primeros componentes del análisis factorial realizado sobre la matriz de datos fisico-químicos de los nueve primeros estanques (se excluyó el estanque $\mathrm{X}$ por apartarse considerablemente de la tendencia general). Con el fin de ofrecer una visión mas clara de los resultados, se ha definido el espacio ocupado por aquellas muestras que aparecen agrupadas, mediante la representación gráfica del valor medio y desviación típica de sus respectivas coordenadas en los tres primeros componentes. Se representan aisladamente las muestras que se desvían de la tendencia general.

La tendencia de variación sigue una pauta estacional, regulada por la temperatura. Su estructura temporal está caracterizada por elevadas concentraciones de silicatos y nitratos en invierno, un gran desarrollo de fitoplancton en primavera, un alto grado de mineralización e intensos procesos de reducción y de descomposición de materia orgánica en verano, y una tendencia a recuperar las condiciones originales de invierno, en otoño (VELASCO, 1989).
Perturbaciones en el medio producen algunas desviaciones de esta tendencia general. Así, en el estanque V, la adición de nutrientes conlleva un rápido e intenso desarrollo de fitoplancton en los meses fríos, al comienzo del estudio y a principios de primavera (muestras D5, E5, F5, M5 y A5); mientras que en el estanque VIII, en los meses de Marzo y Abril (muestras M8 y A8), al final de la primera fase, cuando está sometido a un régimen temporal, presenta característicasfisico-químicas típicas de verano, ya que aumenta considerablemente la mineralización conforme va disminuyendo el volumen de agua. Tras llenarse de nuevo, en Agosto (muestra AG8), presenta una composición fisico-química característica de los meses de primavera, aunque sin un elevado desarrollo de fitoplancton, tendiendo, en los meses siguientes, hacia las característicasde invierno (muestras S8, N8 y 08).

Los rangos de variación temporal de los parámetros fisicoquímicos analizados en los nueve primeros estanques (VELASCO, 1986), están dentro de los límites de tolerancia de los organismos que colonizan estos medios (especies adaptadas a vivir en un amplio rango de condiciones ambientales), por lo que no deben de ejercer limitación para el establecimiento de las poblaciones de insectos acuáticos.

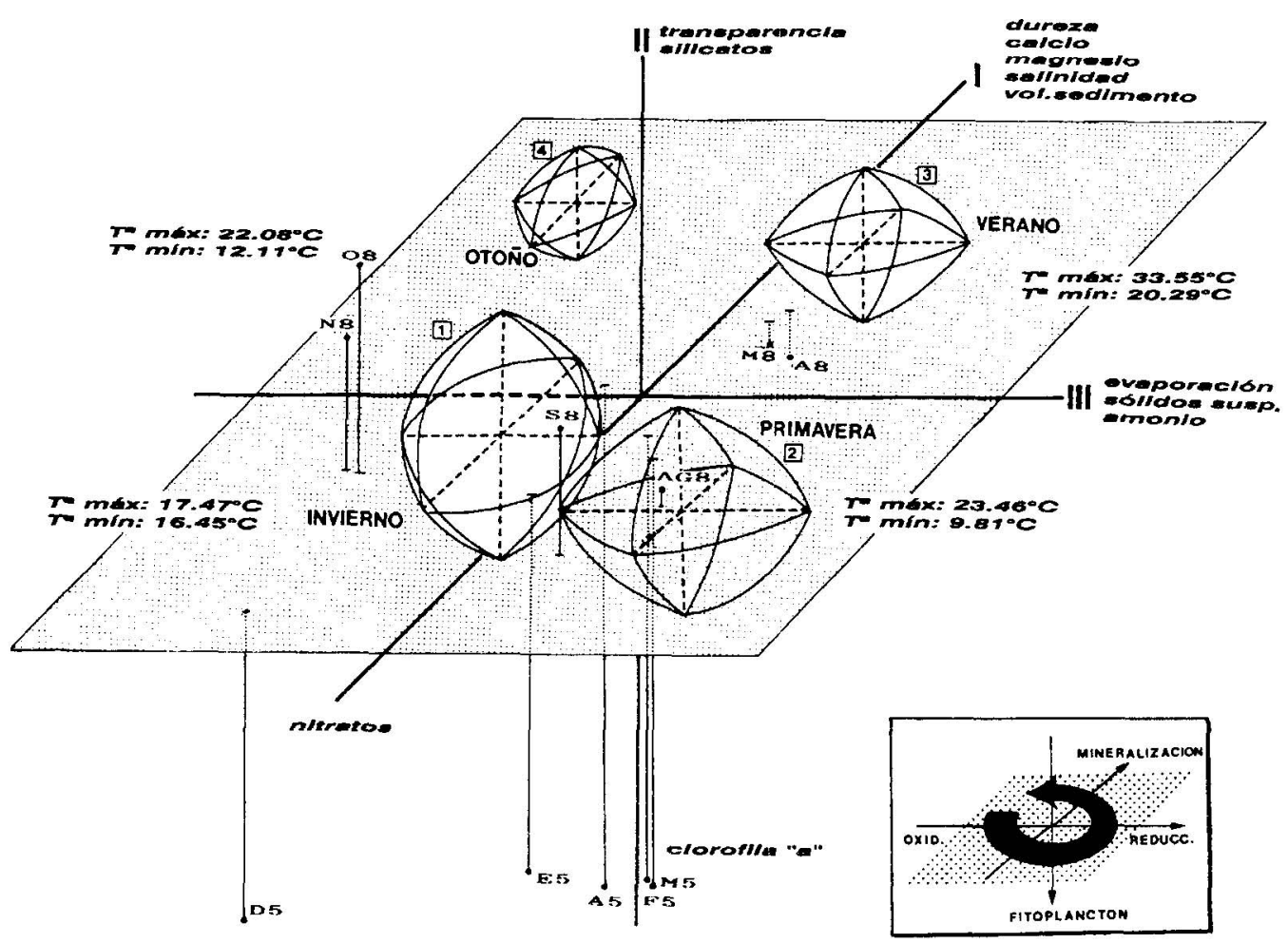

FIGURA 2: Ordenación de las muestras fisico-químicas del agua de los nueve primeros estanques en el espacio definido por los tres primeros componentes del Análisis Multifactorial. Interpretaciónde los componentes y tendencia de variación temporal.

FIGURE 2: Ordering of physico-chemical samples of water from the nine first ponds on the space defined by the three first components of the Factor Analysis. Components interpretation and tendency of temporal variation. 
En cambio, el estanque $\mathrm{X}$ mantiene durante todo el ciclo, aunque con fuertes oscilaciones, una elevada concentración de sales disueltas en el agua (entre 19 y $78 \mathrm{~g} / \mathrm{l}$ ), que confiere a sus aguas una serie de características que lo diferencian del resto de los estanques: registra las temperaturas del agua más altas y una menor tasa de evaporación, presenta las concentraciones de sólidos en suspensión más elevadas, alcanza las mínimas concentraciones de oxígeno disuelto en el agua y limita el desarrollo de las comunidades de algas fitoplanctónicas. Por tanto, es de esperar que el fuerte estrés ambiental producido por la alta salinidad del agua (WILLIAMS, 1985) afecte la colonización y sucesión del estanque, limitando el establecimiento de los organismos que no estén especialmente adaptados a sobreviviren tales condiciones.

\section{El medio biótico: las comunidades de insectos acuáticos}

\section{Lista faunística}

Las comunidades de los estanques están constituidas básicamente por las poblaciones de insectos, aunque estos también fueron colonizados, en menor medida, por otros taxones representantes del orden Crustáceos (Copépodos y Ostrácodos) y del orden Arácnidos (Hydrácaros).

En total se han registrado 40 especies de insectos, pertenecientes a los ordenes Ephemeroptera, Odonata, Heteroptera, Coleoptera y Diptera, que a continuación se relacionan:

\section{INSECTA}

\section{O. EPHEMEROPTERA}

\section{F. BAETIDAE}

1. Cloeon dipterum (Linnaeus, 1761)

2. Procloeon bifidum (Bengtsson, 1912)

F. CAENIDAE

3. Caenis luctuosa (Burmeister, 1839)

O. ODONATA

SO. ANISOPTERA

F. LIBELLULIDAE

4. Orthetrum cancellatum (Linnaeus, 1758)

5. Crocothemis erythraea (Brulle, 1832)

\section{O. HETEROPTERA}

SO. NEPOMORPHA

F. CORIXIDAE

6. Heliocorisa vermiculata (Puton, 1874)

7. Sigara lateralis (Leach, 1817)

\section{F. NOTONECTIDAE}

8. Anisops debilisperplexa Poisson, 1929

9. Anisops sardea Hemch-Schaeffer, 1850
F. VELIIDAE

10. Microvelia pygmaea (Dufour, 1833)

O. COLEOPTERA

SO. HYDRADEPHAGA

F. HALIPLIDAE

11. Haliplus lineatocollis Marsham, 1802

F. DYTISCIDAE

12. Hydroglyphus pusillus (Fabricius, 1781)

13. Potamonectes mariae (Millán \& Rocchi, 1991)

14. Agabus sp.

SO. PALPICORNIA

F. HYDROPHILIDAE

15. Helochares lividus (Forst., 1771)

F. HYDRAENIDAE

16. Ochthebius meridionalis (Rey, 1885)

O. DIPTERA

F. TIPULIDAE

17. Tipula sp.

F. CULICIDAE

18. Culiseta longeareolata (Macquart, 1838)

19. Culex pipiens pipiens Linnaeus, 1758

F. CHIRONOMIDAE

SF. Tanypodinae

20. Procladius sagittalis (Kieffer)

SF. Orthocladiinae

21. Orthocladiinae sp. 1

22. Cricotopus sylvestris (Fabricius, 1794)

23. Cricotopus sp. 2

24. Psectrocladius barbimanus Edwards, 1859

25. Psectrocladius limbatellus (Holmgrem, 1869)

SF. Chironominae

Tribu Chironornini

26. Chironomini sp. 1

27. Chironomus riparius (Meigen, 1818)

28. Chironomus inermifroms (Goetghebuer, 1810)

29. Polypedilum laetum (Meigen, 1818)

30. Polypedilum pullum (Zetterst, 1805)

31. Polypedilum scalaenum (Schamk, 1803)

Tribu Tanytarsini

32. Tanytarsini sp. 1

33. Cladotanytarsus atridorsum Kieffer, 1856

34. Cladotanytarsus mancus Walker, 1875

35. Micropsectra atrofasciata (Kieffer, 1856)

36. Tanytarsus ejuncidus Walker, 1875

37. Tanytarsus sp. 2

F. CERATOPOGONIDAE

38. Dasyhelea sp. 1

39. Dasyhelea sp. 2

F. EPHYDRIDAE

40. Ephydra sp. 


\section{Composición y riqueza específica}

En la Tabla 1 se presenta, para cada estanque, su composición específica, relacionándose los distintos estados del ciclo de vida, así como el número total de las especies pertenecientes a la comunidad acuática. Aparecen indicados con un asterisco aquellos taxones con imagos aéreos, que únicamente se han encontrado en ese estado, y que no forman parte de la comunidad acuática.

Los estanques IV y IX, son los que presentan la mayor riqueza de especies (20), seguidos por los estanques VI (19),
III, V y VII (18). La comunidad de insectos acuáticos más simple es la del estanque $\mathrm{X}$, constituida por una sola especie Ephydra sp., cuyas larvas y pupas son habitantes típicos de aguas salinas litorales y continentales (BERTRAND,1954; MACAN, 1975) gracias a su gran capacidad de osmorregulación (WILLIAMS, 1985). Como era de esperar, la alta salinidad del agua de este estanque impide la colonización de especies que no están adaptadas a fuertes gradientes osmóticos.

De igual forma, el estanque VIII durante su primer llenado, solo es colonizado por tres especies de Dipteros debido a la

TABLA 1: Relación de especies de insectos acuáticos y sus diferentes estados de vida para cada estanque.L, larva; N, ninfa; $\mathrm{P}$, pupa; A, adulto; E, exuvia ninfal; +, encontrado muerto; * sólo el estado aéreo adulto.

TABLE 1: List of species wiht life stages and total species richness for aquatic insects found in each pond. L, larva; N, nymph; P, pupa; A, adult; E, nymphal exuvie; +, found dead; ${ }^{*}$, only aerial adults.

\begin{tabular}{|c|c|c|c|c|c|c|c|c|c|c|c|}
\hline \multicolumn{12}{|c|}{ ESTANQUES } \\
\hline TAXONES & I & II & III & IV & $\mathbf{V}$ & VI & VII & VIII(1) & VIII(2) & IX & $\mathbf{x}$ \\
\hline C.dipterum & $\mathrm{N}, \mathrm{A}$ & $\mathrm{N}, \mathrm{A}$ & $\mathrm{N}, \mathrm{A}$ & $\mathrm{N}, \mathrm{A}$ & $\mathrm{N}, \mathrm{A}$ & $\mathrm{N}, \mathrm{A}$ & $\mathrm{N}, \mathrm{A}$ & & $\mathrm{N}, \mathrm{A}$ & $\mathrm{N}, \mathrm{A}$ & $\mathrm{A}+*$ \\
\hline P.bifidum & & & & & & & & & & & $A+*$ \\
\hline C.luctuosa & & L,A & $\mathrm{L}, \mathrm{A}$ & $\mathrm{L}, \mathrm{A}$ & L,A & & & & & L,A & \\
\hline O.cancellatum & & & $\mathrm{L}, \mathrm{A}$ & $\mathrm{L}, \mathrm{A}$ & & & $\mathrm{L}$ & & & & \\
\hline C.erythraea & & & & $\mathrm{L}$ & $\mathrm{L}$ & & & & & & \\
\hline H.vermiculata & & $\mathrm{L}, \mathrm{A}$ & A & A & A & A & $\mathrm{L}, \mathrm{A}$ & & L,A & $\mathrm{L}, \mathrm{A}$ & \\
\hline S.lateralis & & & & & & $\mathrm{L}, \mathrm{A}$ & A & & & & \\
\hline A.d.-perplexa & & & & A & $\mathrm{L}, \mathrm{A}$ & A & & & $\mathrm{A}$ & A & \\
\hline A.sardea & A & & A & & & & A & & & & \\
\hline M.pygmaea & & & & A & A & & & & & A & \\
\hline H.lineatocollis & & & $\mathrm{L}$ & $\mathrm{L}$ & & & & & & & \\
\hline H.pusillus & & & & L,A & $\mathrm{L}, \mathrm{A}$ & L,A & $\mathrm{A}$ & & & $\mathrm{L}, \mathrm{A}$ & \\
\hline P.mariae & A & & & & & & & & & & \\
\hline Agabus sp. & $\mathrm{L}$ & & & & & & & & & & \\
\hline H.lividus & $\mathrm{L}$ & $\mathrm{L}, \mathrm{A}$ & & & $\mathrm{L}, \mathrm{A}$ & A & & & & $\mathrm{A}$ & \\
\hline O.meridionalis & & $\mathrm{L}$ & & & & $\mathrm{A}$ & & & & $\mathrm{L}$ & \\
\hline Tipula sp. & $\mathrm{L}$ & $\mathrm{L}$ & & & & & & & & & \\
\hline C.longeareolata & $\mathrm{L}, \mathrm{P}, \mathrm{A}$ & $\mathrm{L}, \mathrm{P}, \mathrm{A}$ & L,P,A & L,P,A & $\mathrm{L}, \mathrm{P}, \mathrm{A}$ & L,P,A & L,P,A & $\mathrm{L}, \mathrm{P}$ & $\mathrm{L}, \mathrm{P}, \mathrm{A}$ & L,P, & \\
\hline C.p. pipiens & & & & L & & L,P & $\mathrm{L}$ & & & & \\
\hline P.sagittalis & L,P,A & L,P,A & L,P,A & $\mathrm{L}, \mathrm{P}, \mathrm{A}$ & $\mathrm{L}, \mathrm{P}, \mathrm{A}$ & $L, P, A$ & $\mathrm{~L}, \mathrm{~A}$ & & $\mathrm{~L}, \mathrm{P}, \mathrm{A}$ & L,P, & \\
\hline Orthocladiinae sp.1 & & & & & & & & & $\mathrm{A}^{*}$ & & \\
\hline C.sylvestris & L,P,A & $\mathbf{L}$ & $\mathbf{L}$ & & & $\mathbf{L}$ & $\mathrm{L}$ & & & $\mathbf{L}$ & \\
\hline Cricotopus sp2 & & $\mathrm{L}, \mathrm{P}$ & L,P & $\mathrm{L}, \mathrm{A}$ & $\mathrm{L}$ & & & & & & \\
\hline P.barbimanus & L,P & $\mathrm{L}, \mathrm{A}$ & $\mathrm{L}, \mathrm{P}, \mathrm{A}$ & $\mathrm{A}^{*}$ & $\mathrm{~L}, \mathrm{P}$ & L,P & $\mathrm{L}, \mathrm{P}, \mathrm{A}$ & & $\mathbf{L}$ & L,P & \\
\hline P.limbatellus & & & L,A & $\mathrm{L}, \mathrm{P}, \mathrm{A}$ & & & & & & & \\
\hline Chironomini spl & & & & & & & & & & $\mathrm{L}$ & \\
\hline C.riparius & L,P,A & $\mathbf{L}$ & L,A & L,A & $\mathrm{L}, \mathrm{P}, \mathrm{A}$ & L,P & L,P,A & $\mathbf{L}$ & $\mathrm{A}^{*}$ & $\mathrm{~L}$ & $\mathrm{~A}+*$ \\
\hline C.inermifroms & & & & & $\mathrm{A}^{*}$ & & & & & & \\
\hline P.laetum & & L,P,A & $\mathrm{L}, \mathrm{A}$ & L,P,A & $\mathrm{L}, \mathrm{P}, \mathrm{A}$ & $\mathrm{L}, \mathrm{P}, \mathrm{A}$ & $\mathrm{L}, \mathrm{A}$ & & $\mathrm{L}, \mathrm{P}, \mathrm{A}$ & $\mathrm{L}, \mathrm{P}$ & \\
\hline P.pullum & $\mathrm{L}, \mathrm{P}, \mathrm{A}$ & & & & & & & & & & \\
\hline P.scalaenum & & & & & & & & & & $\mathrm{A}^{*}$ & \\
\hline Tanytarsini sp.1 & & & & & $\mathrm{A}^{*}$ & $\mathrm{~A}^{*}$ & & & $\mathrm{~A}^{*}$ & & \\
\hline C.atridorsum & L,P,A & L,P,A & L, A & $\mathrm{L}, \mathrm{P}, \mathrm{A}$ & $\mathrm{L}, \mathrm{P}$ & L,P,A & $\mathrm{L}, \mathrm{A}$ & & & $\mathrm{L}, \mathrm{P}$, & $\mathrm{A}+*$ \\
\hline C.mancus & $\mathrm{L}, \mathrm{P}, \mathrm{A}$ & & & & & & & & & & \\
\hline M.atrofasciata & $\mathrm{A}^{*}$ & & & & & & & & & & \\
\hline T.ejuncidus & $\mathrm{L}, \mathrm{P}, \mathrm{A}$ & $\mathrm{L}, \mathrm{P}, \mathrm{A}$ & L,A & L,P,A & $\mathrm{L}, \mathrm{A}$ & $\mathrm{L}, \mathrm{P}, \mathrm{A}$ & $\mathrm{L}$ & & $\mathrm{A}^{*}$ & L,P, & \\
\hline Tanytarsus sp2 & & L,P,A & L,P,A & L,P,A & $\mathrm{L}, \mathrm{P}, \mathrm{A}$ & L,P,A & $\mathrm{L}, \mathrm{P}, \mathrm{A}$ & & $\mathrm{L}, \mathrm{P}, \mathrm{A}$ & L,P, & \\
\hline Dasyhelea spl & L,P & $\mathrm{L}, \mathrm{P}, \mathrm{A}$ & $\mathrm{L}, \mathrm{P}$ & $\mathrm{L}, \mathrm{P}$ & $\mathrm{L}, \mathrm{P}$ & $\mathrm{L}, \mathrm{P}$ & $\mathrm{L}, \mathrm{P}$ & $\mathrm{L}, \mathrm{P}$ & & L,P, & \\
\hline Dasyhelea sp2 & & & & & & $\mathrm{L}$ & $\mathrm{L}, \mathrm{P}$ & & $\mathrm{L}, \mathrm{P}, \mathrm{A}$ & $\mathrm{L}$ & \\
\hline Ephydra sp. & & & & & $\mathrm{A}$ & & & & & $A^{*}$ & ,A,P \\
\hline$N^{\circ}$ DE ESPECIES & 15 & 17 & 18 & 20 & 18 & 19 & 18 & 3 & 10 & 20 & 1 \\
\hline
\end{tabular}


fuerte limitación que ejerce la temporalidad del medio para la colonización y asentamiento de las mismas; siendo colonizado posteriormente, durante el régimen permanente, por diez especies, siendo Culiseta longeareolata la única especie común en las dos etapas.

En general, salvo en el estanque $\mathrm{X}$, no se observan grandes diferencias en la composición faunística de los estanques. Cloeon dipterum, Culiseta longeareolata, Procladius sagittalis, Chironomus riparius y Dasyhelea sp.1 son comunes en los nueve primeros estanques. En cambio, otras especies son características o sólo se desarrollan en uno de los estanques: Polypedilum pullum, Potamonectes mariae y Agabus sp. (estanque I); Cladotanytarsus mancus (estanque VIII); y la mencionada Ephydra sp. (estanque X).

Cuatro estanques tienen representantes de todos los órdenes de insectos encontrados (III, IV, V y VII). Efemerópteros, Heterópteros y Dípteros conjuntamente, están presentes en todos los estanques, excepto en el X. Los Coleópteros colonizan todos los estanques salvo el VIII y X. Los Odonatos, por el contrario, sólo aparecen en los estanques III, IV, V y VII.

El orden que más contribuye a la riqueza de especies, en todos los estanques, es el de los Dípteros, y dentro de ellos, la familia Chironomidae con, aproximadamente, la mitad del total de las especies. El resto de familias de Dípteros y de los restantes órdenes contribuyen al espectro de riqueza con una o dos especies como máximo.

\section{Frecuencia y dominancia}

En todos los estanques, los Dípteros son el orden más abundante en cuanto a número de individuos. Dentro de estos, los Quironómidos representan, aproximadamente, la mitad del total de individuos. La importancia numérica de esta familia es debida, fundamentalmente, al Tanypodino Procladius sagittalis, que es la especie más abundante en todos los estanques (salvo en el VII y X), llegando a representar el $57.41 \%$ del número total de individuos en el estanque IX (ver Apéndice). El resto de especies de Quironómidos contribuyen en menor grado, aunque algunos Tanytarsinos tienen una importancia considerable, como ocurre con Tanytarsus sp.2 en el estanque V (25.10\%), Tanytarsus ejuncidus en el estanque IV (15.91\%) y Cladotanytarsus mancus en el estanque VIII, durante la segunda etapa ( $16.61 \%)$.

Los Culícidos, representados fundamentalmente por Culiseta longeareolata, son la segunda familia de Dípteros en importancia numérica y su porcentaje varía desde $5.80 \%$ (estanque III) hasta 99\% (estanque VIII, $1^{\text {cr }}$ periodo). El bajo número de individuos registrado en los estanques II y III pare- ce estar motivado por la presencia de los macrófitos Cladophora sp. y Chara vulgaris, que inhiben de varios modos su desarrollo larvario (CABALLERO, 1919; PARDO, 1923; HUTCHINSON, G.E. 1981).

Dentro de los Efemerópteros, Cloeon dipterum, constituye otra de las poblaciones básicas de los estanques, oscilando sus valores de dominancia de $23.48 \%$ (estanque III) a $1.20 \%$ (estanque VI). Los Heterópteros sólo tienen una importancia numérica considerable en los estanques II, VI y VII, debido a los Coríxidos Heliocorisa vermiculata (II y VII) y Sigara late ralis (VI). Entre las especies de Coleópteros, sólo el Dytíscido Hydroglyphus pusillus presenta una abundancia relativa superior al $1 \%$, en los estanques V y VI. Igual sucede con Orthetrum cancellatum en el estanque III, dentro de Odonatos.

La baja contribución de la mayoría de las especies de Heterópteros y Coleópteros al número total de individuos, se debe a que, normalmente, sus poblaciones constan de unos pocos individuos adultos que utilizan estos medios para alimentarse. Sólo adquieren una abundancia relativamente importante aquellas especies que se reproducen sucesivamente en los estanques.

En función de los valores de frecuencia y abundancia relativa, se han clasificado las distintas poblaciones que componen las comunidades de insectos acuáticos de cada estanque (Tabla 2) según las categorías propuestas por THIERY (1978), con rangos de valores adaptados al presente estudio y que se especifican en dicha tabla. Como se puede observar, las comunidades de los estanques (salvo los estanques VIII y X) están formadas por un bajo número de especies fundamentales y constantes, y un gran número de especies acompañantes, accesorias y esporádicas, lo que manifiesta la inmadurez de estos sistemas artificiales durante el primer año de su existencia.

Cloeon dipterum, Culiseta longeareolata y Procladius sagittalis son las especies fundamentales más comunes en los estanques. Son especies con ciclos de vida polivoltinos, que desarrollan varias generaciones en los estanques, lo que les permite mantener altas densidades de población durante la mayor parte del periodo de estudio. Dentro de este grupo, también destaca Heliocorisa vermiculata, en los estanques II y VII, Sigara lateralis en el estanque VI y Tanytarsus sp.2 en el V. En el estanque VIII, durante el primer periodo de colonización, Culiseta longeareolata es la única especie fundamental, mientras que en el segundo periodo, su puesto es ocupado por Polypedilum laetum y Cladotanytarsus mancus junto con Procladius sagittalis.

Las especies constantes, al igual que las fundamentales, se presentan en más del 50\% de las muestras, pero se diferencian por la dominancia, con valores siempre menores al 10\%. Es el 
'TABLA 2: Clasificación de las especies segun su valor de frecuencia y abundancia relativa (categorias modificadas de THIERY, 1978).

TABLE 2: Classification of species in terms of frequency and relative abundance categories (modified from THIERY, 1978).

ESPECIES

\begin{tabular}{|c|c|c|c|c|c|}
\hline ESTANQUES & $\begin{array}{l}\text { FUNDAMENTALES } \\
\mathbf{F}>\mathbf{5 0} \%, \mathbf{D}>\mathbf{1 0} \%\end{array}$ & $\begin{array}{l}\text { CONSTANTES } \\
\mathbf{F}>\mathbf{5 0} \%, \mathbf{D}<\mathbf{1 0} \%\end{array}$ & $\begin{array}{l}\text { ACOMPAÑANTES } \\
20 \%>\mathbf{F}<50 \%, \mathrm{D}<10 \%\end{array}$ & $\begin{array}{l}\text { ACCESORIAS } \\
\mathbf{6 \%}>\mathbf{F}<\mathbf{2 0} \%, \mathrm{D}<\mathbf{1 0} \%\end{array}$ & $\begin{array}{l}\text { ESPORADICAS } \\
\mathbf{F}<\mathbf{6 \%}, \mathrm{D}<10 \%\end{array}$ \\
\hline $\bar{I}$ & $\begin{array}{l}\text { C.dipterum } \\
\text { C.Iongeareolata } \\
\text { P.sagittalis }\end{array}$ & $\begin{array}{l}\text { C.riparius } \\
\text { C.atridorsum }\end{array}$ & $\begin{array}{l}\text { Tipula sp. } \\
\text { P.pullum } \\
\text { T.ejuncidus } \\
\text { Dasyhelea spl } \\
\end{array}$ & $\begin{array}{l}\text { C.sylvestris } \\
\text { P.barbimanus }\end{array}$ & $\begin{array}{l}\text { A.sardea } \\
\text { P.mariae } \\
\text { Agabus sp. } \\
\text { H.lividus }\end{array}$ \\
\hline II & $\begin{array}{l}\text { C.dipterum } \\
\text { H.vermiculata } \\
\text { P.sagittalis }\end{array}$ & C.longeareolata & $\begin{array}{l}\text { H.lividus } \\
\text { C.riparius } \\
\text { P.laetum } \\
\text { C.atridorsum } \\
\text { Tanytarsus sp2 } \\
\text { Dasyhelea sp1 } \\
\end{array}$ & $\begin{array}{l}\text { C.sylvestris } \\
\text { Cricotopus sp2 } \\
\text { P.barbimanus } \\
\text { T.ejuncidus }\end{array}$ & $\begin{array}{l}\text { C.luctuosa } \\
\text { Tipula sp. } \\
\text { O.meridionalis }\end{array}$ \\
\hline$\overline{\text { III }}$ & $\begin{array}{l}\text { C.dipterum } \\
\text { P.sagittalis }\end{array}$ & C.longeareolata & $\begin{array}{l}\text { C.luctuosa } \\
\text { O.cancellatum } \\
\text { H.lineatocollis } \\
\text { Cricotopus sp2 } \\
\text { P.barbimanus } \\
\text { C.riparius } \\
\text { P.laetum } \\
\text { Tanytarsus sp2 }\end{array}$ & $\begin{array}{l}\text { A.sardea } \\
\text { C.atridorsum }\end{array}$ & $\begin{array}{l}\text { H.vermiculata } \\
\text { P.limbatellus } \\
\text { T.ejuncidus } \\
\text { Dasyhelea spl }\end{array}$ \\
\hline$\overline{I V}$ & $\begin{array}{l}\text { C.dipterum } \\
\text { C.longeareolata } \\
\text { P.sagittalis }\end{array}$ & & $\begin{array}{l}\text { C.luctuosa } \\
\text { O.cancellatum } \\
\text { H.pusillus } \\
\text { P.limbatellus } \\
\text { C.riparius } \\
\text { P.laetum } \\
\text { C.atridorsum } \\
\text { Tanytarsus sp2 } \\
\end{array}$ & $\begin{array}{l}\text { C.erythraea } \\
\text { Dasyhelea sp1 }\end{array}$ & $\begin{array}{l}\text { H.vermiculata } \\
\text { A.d.perplexa } \\
\text { M.pygmaea } \\
\text { H.lineatocollis } \\
\text { C.p.pipiens } \\
\text { Cricotopus sp2 }\end{array}$ \\
\hline $\mathrm{V}$ & $\begin{array}{l}\text { C.longeareolata } \\
\text { P.sagittalis } \\
\text { Tanytarsus sp2 }\end{array}$ & C.dipterum & $\begin{array}{l}\text { C.luctuosa } \\
\text { C.erythraea } \\
\text { A.d.perplexa } \\
\text { H.pusillus } \\
\text { Cricotopus sp2 } \\
\text { P.barbimanus } \\
\text { C.riparius } \\
\text { P.laetum } \\
\text { C.atridorsum }\end{array}$ & $\begin{array}{l}\text { H.lividus } \\
\text { T.ejuncidus } \\
\text { Dasyhelea sp1 }\end{array}$ & $\begin{array}{l}\text { H.vermiculata } \\
\text { M.pygmaea } \\
\text { Ephydra sp. }\end{array}$ \\
\hline$\overline{\mathrm{VI}}$ & $\begin{array}{l}\text { S.lateralis } \\
\text { C.longeareolata } \\
\text { P.sagittalis }\end{array}$ & C.dipterum & $\begin{array}{l}\text { H.pusillus } \\
\text { P.barbimanus } \\
\text { P.laetum } \\
\text { C.atridorsum } \\
\text { T.ejuncidus } \\
\text { Tanytarsussp.2 }\end{array}$ & $\begin{array}{l}\text { H.vermiculata } \\
\text { C.sylvestris } \\
\text { C.riparius }\end{array}$ & $\begin{array}{l}\text { A.d.perplexa } \\
\text { C.p.pipiens } \\
\text { Dasyhelea sp1 } \\
\text { Dasyhelea sp2 }\end{array}$ \\
\hline VII & $\begin{array}{l}\text { C.dipterum } \\
\text { H.vermiculata } \\
\text { C.longeareolata } \\
\text { P.sagittalis }\end{array}$ & & $\begin{array}{l}\text { P.barbimanus } \\
\text { C.riparius } \\
\text { P.laetum } \\
\text { Tanytarsus sp. } 2\end{array}$ & $\begin{array}{l}\text { O.cancellatum } \\
\text { A.sardea } \\
\text { H.pusillus } \\
\text { C.atridorsum } \\
\text { T.ejuncidus } \\
\text { Dasyhelea spl } \\
\text { Dasyhelea sp2 } \\
\end{array}$ & $\begin{array}{l}\text { S.lateralis } \\
\text { C.p.pipiens } \\
\text { C.sylvestris }\end{array}$ \\
\hline VIII(1) & C.longeareolata & & C.riparius & Dasyhelea spl & P.barbimanus \\
\hline VIII(2) & $\begin{array}{l}\text { P.sagittalis } \\
\text { P.laetum } \\
\text { C.mancus }\end{array}$ & $\begin{array}{l}\text { C.longearolata } \\
\text { C.dipterum } \\
\text { Tanytarsus sp2 }\end{array}$ & $\begin{array}{l}\text { Dasyhelea sp2 } \\
\text { H.vermiculata } \\
\text { A.d.-perplexa }\end{array}$ & P.barbimanus & \\
\hline IX & $\begin{array}{l}\text { C.longeareolata } \\
\text { P.sagittalis }\end{array}$ & C.dipterum & $\begin{array}{l}\text { H.vermiculata } \\
\text { H.pusillus } \\
\text { P.barbimanus } \\
\text { P.laetum } \\
\text { C.atridorsum } \\
\text { T.ejuncidus } \\
\text { Tanytarsus sp2 }\end{array}$ & $\begin{array}{l}\text { C.luctuosa } \\
\text { Dasyhelea spl }\end{array}$ & $\begin{array}{l}\text { A.d.perplexa } \\
\text { M.pygmaea } \\
\text { H.lividus } \\
\text { O.meridionalis } \\
\text { C.sylvestris } \\
\text { Chironominisp I } \\
\text { C.riparius } \\
\text { Dasyheleasp2 } \\
\text { Ephydra so. }\end{array}$ \\
\hline
\end{tabular}


caso de Culiseta longeareolata en los estanques II y III, donde a pesar de desarrollar varias generaciones al año, no consiguen dejar gran número de descendientes.

Dentro de los grupos de especies acompañantes y accesorias, aparecen aquellas poblaciones que desarrollan su ciclo de vida en los estanques, incluso más de una generación, pero durante un cono espacio de tiempo. El gran número de especies de este tipo en dichos ambientes, es un índice de la alta tasa de sustitución de especies durante las primeras etapas en la colonización de medios recién creados.

En la categoría de esporádicas se encuentran aquellas especies que, generalmente, aparecen en muy bajo número y en una o dos muestras como máximo. La mayoría son Coleópteros y Heterópteros que se han registrado única y aisladamente en los estanques, en forma de adultos, utilizando estos cuerpos de agua como medios transitorios de alimentación en sus desplazamientos. El resto de especies de este grupo son Dípteros, que aunque ovopositan en los estanques (hay registro de larvas), no llegan a completar su ciclo de vida.

Por último, se presentan algunos casos que quedan fuera de esta clasificación general. Se trata de especies dominantes de claro carácter estacional $(\mathrm{F}<50 \%, \mathrm{D}>10 \%)$, como es el caso de Cricotopus sylvestris (estanque III) y Tanytarus ejuncidus (estanque IV) cuyas poblaciones llegan a ser dominantes pero sólo en determinados momentos del periodo de estudio.

\section{Diversidad y equitabilidad}

En la Tabla 3 aparece, junto al número de especies y número total de individuos de cada estanque, sus valores de diversidad, diversidad máxima y equitabilidad. El estanque II, con 17 especies (muy pocas accesorias y esporádicas) y el número de individuos más bajo de los registrados tras los estanques $\mathrm{X}$ y

TABLA 3: Riqueza de especies, número total de individuos, diversidad, diversidad máxima y equitabilidad de las comunidades de insectos acuáticos de cada estanque.

TABLE 3: Species richness, total number of individuals, diversity, maximum diversity, and evenness of aquatic insect communitiesin each pond.

\begin{tabular}{lrrrrr} 
ESTANQUE & N'sp $^{\circ}$ & Noindv. & H' & Hmáx. & E \\
\hline I & 15 & 57555 & 2.19 & 3.91 & 0.56 \\
II & 17 & 23608 & 2.69 & 4.09 & 0.66 \\
III & 18 & 31397 & 2.64 & 4.17 & 0.63 \\
IV & 20 & 50426 & 2.56 & 4.32 & 0.59 \\
V & 18 & 95351 & 2.41 & 4.25 & 0.57 \\
VI & 19 & 34426 & 2.43 & 4.25 & 0.54 \\
VII & 18 & 49643 & 2.33 & 4.17 & 0.56 \\
VIII(1) & 3 & 7368 & 0.04 & 0.33 & 0.04 \\
VII(2) & 10 & 48243 & 1.76 & 2.67 & 0.64 \\
IX & 20 & 68044 & 1.69 & 4.39 & 0.38 \\
X & 1 & 653 & 0.00 & 0.00 & 0.00 \\
\hline
\end{tabular}

VIII, presenta los valores de diversidad y equitabilidad más altos $(\mathrm{H}=2.69$ y $\mathrm{E}=0.65)$. En cambio, el estanque IX, con la máxima riqueza específica, pero con un elevado número de especies esporádicas, presenta valores de diversidad y equitabilidad bastante bajos $(\mathrm{H}=1.69$ y $\mathrm{E}=0.38)$ en comparación con el resto de los estanques. Su diversidad es sensiblemente menor a la del estanque IV, con igual número de especies, e incluso mas baja que la del estanque VIII durante su segunda etapa.

El estanque $\mathrm{V}$ es el que soporta el mayor número de individuos, aunque no de especies, presentando valores de diversidad y equitabilidad semejantes al resto de estanques $(\mathrm{H}=2.41 \mathrm{y}$ $\mathrm{E}=0.57$ ).

\section{DISCUSIÓN}

Las especies de insectos acuáticos que colonizan los estanques son típicos habitantes de los humedales del sureste español (RAMÍ́REZ-DÍAZ et al. 1992), así como de las ramblas del sector más árido (ORTEGA, et al. 1991). Aunque ningún organismo se puede definir totalmente como de la selección "r", " Ko "A" (PIANKA, 1970), la mayoría de estas especies pueden ser consideradas en sentido amplio, como estrategas de la " $r$ ", estrategia típica en medios inestables y en las etapas pioneras de la sucesión de los ecosistemas (MARGALEF, 1977). Presentan un carácter oportunista y eurioico, definido por su capacidad de dispersión activa, reproducción temprana en su ciclo de vida, elevada fecundidad y tasa de desarrollo, alimentación generalista, poca capacidad competitiva y tolerancia a un amplio rango de condiciones ambientales; todo ello, ligado a una vida breve, que les permite colonizar rápidamente cualquier medio nuevo o perturbado.

La colonización y asentamiento de las especies, en los estanques estudiados, depende, aparte del factor azar, de propiedades intrínsecas de cada especie, como su euricidad, capacidad de dispersión, poder de multiplicación, competitividad, selección de lugares de ovoposición, requerimientos de las larvas, etc.; así como de otros factores locales, como la acción de estresores ambientales, la disponibilidad de alimento y la presencia de especies competidoras y/o depredadoras (VELASCO, 1989).

Culiseta longeareolata, Procladius sagittalis, Chironomus riparius, Dasyhelea spl y Cloeon dipterum, son las especies más eurioicas y con mayor exito colonizador, ya que están presentes en nueve de los diez medios estudiados. En cambio, Haliplus lineatocollis, Potamonectes mariae, Agabus sp, Polypedilum pullum, Cladotanytarsus mancus y Ephydra sp, son características de uno sólo de estos medios.

A pesar de las diferencias en las condiciones microambientales entre los estanques, las comunidades de insectos acuáti- 
cos presentan una composición y estructura bastante similar, salvo en los estanques VIII ( $1^{\text {er }}$ periodo) y X, donde la temporalidad del medio y la alta salinidad del agua, respectivamente, ejercen una fuerte limitación para el establecimiento de las especies.

Los Dípteros, distribuidos en todos los estanques, constituyen el grupo más importante, tanto en riqueza de especies como en abundancia, representando, sólo los Quironómidos, aproximadamente la mitad del número total de especies e individuos. Este hecho era de esperar, teniendo en cuenta la amplia distribución de esta familia en todos los medios acuáticos y su gran riqueza taxonómica (WIGGINS et al. 1980). En cambio, los Odonatos, son el orden de insectos con una distribución más restringida, menor número de especies y una abundancia relativa insignificante, debido en gran parte a la ausencia de vegetación emergente en los medios estudiados, condición indispensable para la invasión de especies con ovoposición endofítica, al igual que para la emergencia de los adultos (BARNES, 1983).

La estructura de las comunidades de insectos acuáticos en los estanques es bastante simple, reflejo de la inmadurez de estos sistemas, donde existe una alta tasa de sustitución de especies durante las etapas pioneras de su sucesión. Las comunidades están constituidas por unas pocas especies "fundamentales" y "constantes", generalmente Cloeon dipterum, Culiseta longeareolata y Procladius sagittalis, con ciclos de vida polivoltinos que mantienen altas densidades de población durante gran parte del año. El resto, son especies "acompañantes", "accesorias" y "esporádicas", presentándose durante un corto espacio de tiempo y con densidades de población más bajas. En la Tabla 4 se especifica la estrategia de vida seguida por cada una de las especies en los estanques estudiados.

TABLA 4: Estrategias de vida de las especies en cada estanque. 1 , no se reproduce; 2 , se reproduce: a, no completa su ciclo de vida, b, completa el ciclo de vida. (U) una generación, $(\mathbf{P})$ varias generaciones.

TABLE 4: Life snategies of species in each pond. 1, non-reproductive; 2, reproductive: a, incomplete life cycle, b, complete life cycle. (U) one generation, (P) several generations.

ESTANQUES

\begin{tabular}{|c|c|c|c|c|c|c|c|c|c|c|c|}
\hline TAXONES & I & II & III & IV & $\mathbf{v}$ & VI & VII & VIII(1) & VIII(2) & IX & $\mathbf{X}$ \\
\hline C.dipterum & $2 b(P)$ & $2 b(P)$ & $2 b(P)$ & $2 b(P)$ & $2 b(P)$ & $2 b(P)$ & $2 b(P)$ & & $2 b(P)$ & $2 b(P)$ & \\
\hline C. luctuosa & & $2 b(U)$ & $2 b(P)$ & $2 b(P)$ & $2 b(P)$ & & & & & $2 b(U)$ & \\
\hline O.cancellatum & & & $2 b(P)$ & $2 b(P)$ & & & $2 \mathbf{a}$ & & & & \\
\hline C.erythraea & & & & $2 a$ & $2 \mathrm{a}$ & & & & & & \\
\hline H.vermiculata & & $2 b(P)$ & 1 & 1 & 1 & 1 & $2 b(P)$ & & $2 a$ & $2 b(U)$ & \\
\hline S.lateralis & & & & & & $2 b(P)$ & 1 & & & & \\
\hline A.d.-perplexa & & & & 1 & $2 \mathrm{~b}(\mathrm{U})$ & 1 & & & 1 & 1 & \\
\hline A.sardea & 1 & & 1 & & & & & & & & \\
\hline M.pygmaea & & & & 1 & 1 & & & & & 1 & \\
\hline H.lineatocollis & & & $2 a$ & $2 a ?$ & & & & & & & \\
\hline H.pusillus & & & & $2 b(P)$ & $2 b(P)$ & $2 b(P)$ & 1 & & & $2 b(P$ & \\
\hline P.mariae & 1 & & & & & & & & & & \\
\hline Agabus sp. & $2 a$ & & & & & & & & & & \\
\hline H.lividus & 2 & $2 b(U)$ & & & $2 b(U)$ & 1 & & & & 1 & \\
\hline O.meridionalis & & $2 a$ & & & & 1 & & & & $2 a$ & \\
\hline Tipula sp. & $2 a$ & $2 a$ & & & & & & & & & \\
\hline C.longeareolata & $2 b(P)$ & $2 b(P)$ & $2 \mathrm{~b}(\mathrm{P})$ & $2 b(P)$ & $2 \mathrm{~b}(\mathrm{P})$ & $2 b(P)$ & $2 \mathrm{~b}(\mathrm{P})$ & $2 b(P)$ & $2 b(P)$ & $2 b(P)$ & \\
\hline C.p. pipiens & & & & & $2 b(U)$ & & $2 b(U)$ & & $2 b(U)$ & & \\
\hline P.sagittalis & $2 b(P)$ & $2 \mathrm{~b}(\mathrm{P})$ & $2 \mathrm{~b}(\mathrm{P})$ & $2 \mathrm{~b}(\mathrm{P})$ & $2 b(P)$ & $2 b(P)$ & $2 \mathrm{~b}(\mathrm{P})$ & $2 b(P)$ & & $2 b(P)$ & \\
\hline C.sylvestris & $2 b(U)$ & $2 a$ & $2 b(P)$ & & & $2 \mathbf{a}$ & $2 a$ & & & $2 \mathrm{a}$ & \\
\hline Cricotopus sp2 & & $2 b(P)$ & $2 b(P)$ & $2 \mathbf{a}$ & $2 a$ & & & & & & \\
\hline P.barbimanus & $2 b(P)$ & $2 \mathrm{~b}(\mathrm{P})$ & $2 b(\mathbf{P})$ & & $2 \mathrm{~b}(\mathrm{P})$ & $2 b(\mathbf{P})$ & $2 b(P)$ & & $2 a$ & $2 b(P)$ & \\
\hline P.limbatellus & & & $2 a$ & $2 b(P)$ & & & & & & & \\
\hline Chironomini sp1 & & & & & & & & & $2 \mathrm{a}$ & & \\
\hline C.riparius & $2 \mathrm{~b}(\mathrm{P})$ & $2 b(P)$ & $2 b(P)$ & $2 b(P)$ & $2 b(P)$ & $2 \mathrm{~b}(\mathrm{P})$ & $2 b(P)$ & $2 b(P)$ & 1 & $2 b(P)$ & \\
\hline P. laetum & & $2 b(P)$ & $2 b(P)$ & $2 b(P)$ & $2 b(P)$ & $2 b(P)$ & $2 b(P)$ & & $2 b(P)$ & $2 b(P)$ & \\
\hline P.pullum & $2 \mathrm{~b}(\mathrm{P})$ & & & & & & & & & & \\
\hline C.atridorsum & $2 b(P)$ & $2 b(P)$ & $2 b(P)$ & $2 b(P)$ & $2 b(P)$ & $2 b(P)$ & $2 b(P)$ & & & $2 b(P)$ & \\
\hline C.mancus & & & & & & & & & $2 b(P)$ & & \\
\hline T.ejuncidus & $2 b(P)$ & $2 b(P)$ & $2 b(P)$ & $2 b(P)$ & $2 b(P)$ & $2 b(P)$ & $2 b(P)$ & & & $2 b(P)$ & \\
\hline Tanytarsus sp2 & & $2 b(P)$ & $2 b(P)$ & $2 b(P)$ & $2 b(P)$ & $2 b(P)$ & $2 b(P)$ & & $2 \mathrm{~b}(\mathrm{P})$ & $2 b(P)$ & \\
\hline Dasyhelea spl & $2 b(P)$ & $2 b(P)$ & $2 \mathrm{~b}(\mathrm{U})$ & $2 b(U)$ & $2 b(U)$ & $2 b(U)$ & $2 b(U)$ & & $2 b(U)$ & $2 b(P)$ & \\
\hline Dasyhelea sp2 & & & & & & $2 b(U)$ & $2 b(U)$ & $2 b(\mathbf{U})$ & & $2 b(U)$ & \\
\hline Ephydra sp. & & & & & & & & & & & $2 b(P)$ \\
\hline
\end{tabular}


Dos tipos básicos de estrategia de vida se dan entre las especies colonizadoras:

1) Especies que utilizan los estanques como medios transitorios en sus vuelos de dispersión, donde se alimentan, pero no se reproducen. A pesar de que interaccionan fuertemente con la comunidad, su importancia numérica es baja, ya que se presentan de forma aislada y puntual. Es el caso de los grandes depredadores adultos de la mayoría de las especies de Coleópteros y Heterópteros acuáticos.

2) Especies que se reproducen u ovopositan en los estanques. Sus poblaciones constituyen los componentes básicos de las comunidades, y son las que permanecen mayor tiempo en ellos. Dentro de este grupo, se distinguen, a su vez, dos tipos:

a) Especies que no llegan a desarrollar el ciclo de vida completo, debido principalmente, a la falta de determinados requerimientos ecológicos y/o a la acción de la competencia interespecífica y la predación. Un claro ejemplo, lo constituyen los
Coleópteros Haliplus lineatocollis y Ochthebius meridionalis, y el Díptero Típula sp, que debido a la estructura de los medios estudiados (paredes verticales de cemento), no pueden realizar la fase de pupación terrestre.

b) Especies que desarrollan el ciclo completo, pudiendo realizar una o varias generaciones anuales. Sus poblaciones juegan un papel muy importante dentro de la comunidad. Son los verdaderos colonizadores con éxito.

En general, las comunidades de insectos están constituidas por un número de especies relativamente bajo comparado con el observado en charcas naturales próximas, donde la máxima riqueza encontrada de insectos acuáticos ha sido 21 especies, sin incluir los Dípteros, que son su principal componente (SUÁREZ et al. 1991). Esta situación es lógica dada la artificialidad y simplicidad de los medios estudiados y su corto periodo de existencia. En cambio los valores de diversidad de los estanques están dentro del rango observado por los autores

APÉNDICE: Frecuencia y abundancia relativa para las diferentes especies de cada estanque.

APPENDIX:Frequency and relative abundance of different species in each pond.

\section{ESTANQUES}

\begin{tabular}{|c|c|c|c|c|c|c|c|c|c|c|c|c|c|c|c|c|c|c|c|c|c|c|}
\hline \multirow[b]{2}{*}{ TAXONES } & \multicolumn{2}{|c|}{ I } & \multicolumn{2}{|c|}{ II } & \multicolumn{2}{|c|}{ III } & \multicolumn{2}{|c|}{ IV } & \multicolumn{2}{|c|}{$\mathbf{V}$} & \multicolumn{2}{|c|}{ VI } & \multicolumn{2}{|c|}{ VII } & \multicolumn{2}{|c|}{ VIII (1) } & \multicolumn{2}{|c|}{ VIII (2) } & \multicolumn{2}{|c|}{ IX } & \multicolumn{2}{|c|}{$\mathbf{X}$} \\
\hline & $\mathrm{F} \%$ & D\% & $\mathbf{F} \%$ & $\mathrm{D} \%$ & $\mathbf{F} \%$ & D\% & $\mathrm{F} \%$ & $\mathrm{D} \%$ & $\mathrm{~F} \%$ & D\% & $\mathbf{F} \%$ & $\mathrm{D} \%$ & $\mathrm{~F} \%$ & $\mathrm{D} \%$ & $\mathbf{F} \%$ & $\mathrm{D} \%$ & F\% & D\% & $\mathbf{F} \%$ & D\% & $\mathrm{F} \%$ & D\% \\
\hline C.dipterum & 52.94 & 1.20 & 0.00 & 17.90 & 64.70 & 23.48 & 70.58 & 18.49 & 64.70 & 8.71 & 52.94 & 1.20 & 58.82 & 14.77 & & & 91.66 & 9.32 & 70.58 & 7.79 & & \\
\hline C. luctuosa & & & 5.88 & 0.80 & 20.58 & 0.71 & 32.35 & 0.92 & 20.58 & 0.13 & & & & & & & & & 11.76 & 0.12 & & \\
\hline O.cancellatum & & & & & 29.41 & 1.55 & 26.47 & 0.44 & & & & & 11.76 & 0.01 & & & & & & & & \\
\hline C.erythraea & & & & & & & 8.82 & 0.00 & 20.58 & 0.09 & & & & & & & & & & & & \\
\hline H.vermiculata & & & 17.05 & 22.00 & 2.94 & 0.00 & 2.94 & 0.00 & 5.88 & 0.00 & 8.82 & 0.00 & 55.88 & 10.06 & & & 25.00 & 0.01 & 20.58 & 0.03 & & \\
\hline S. lateralis & & & & & & & & & & & 55.88 & 3.53 & 2.94 & 0.00 & & & & & & & & \\
\hline Ad -perplexa & & & & & & & 2.94 & 0.00 & 23.52 & 0.32 & 2.94 & 0.00 & & & & & 25.00 & 0.00 & 5.88 & 0.00 & & \\
\hline A.sardea & 2.94 & 0.00 & & & 11.76 & 0.01 & & & & & & & 8.82 & 0.00 & & & & & & & & \\
\hline M.pygmaea & & & & & & & 2.94 & 0.00 & 5.88 & 0.00 & & & & & & & & & 5.88 & 0.00 & & \\
\hline H. lineatocollis & & & & & 20.58 & 0.04 & 2.94 & 0.00 & & & & & & & & & & & & & & \\
\hline H.pusillus & & & & & & & 20.58 & 0.84 & 42.17 & 2.09 & 29.41 & 1.28 & 11.76 & 0.00 & & & & & 26.47 & 0.14 & & \\
\hline P. mariae & 2.94 & 0.00 & & & & & & & & & & & & & & & & & & & & \\
\hline H.lividus & 2.94 & 0.00 & 6.47 & 0.50 & & & & & 11.76 & 0.03 & 29.41 & 0.00 & & & & & & & 2.94 & 0.00 & & \\
\hline O.meridionalis & & & 2.94 & 0.00 & & & & & & & 29.41 & 0.00 & & & & & & & 2.94 & 0.00 & & \\
\hline Tripula sp. & 0.58 & 0.11 & 5.88 & 0.00 & & & & & & & & & & & & & & & & & & \\
\hline C.longeareolata & 4.70 & 22.09 & 8.82 & 7.60 & 52.94 & 5.80 & 61.76 & 16.49 & 82.35 & 16.07 & 99.41 & 27.34 & 76.47 & 44.31 & 91.66 & 99.00 & 83.33 & 6.28 & 91.17 & 28.42 & & \\
\hline C.p. pipiens & & & & & & & 2.94 & 0.24 & & & 5.08 & 0.55 & 2.94 & 0.82 & & & & & & & & \\
\hline P.sagittalis & 4.70 & 48.08 & 7.64 & 35.30 & 64.70 & 37.95 & 64.70 & 37.07 & 67.64 & 39.19 & 61.76 & 39.56 & 67.64 & 21.41 & & & 83.33 & 50.77 & 73.52 & 57.41 & & \\
\hline C.sylvestris & 8.82 & 0.06 & 4.70 & 0.50 & 5.88 & 11.47 & & & & & 11.76 & 0.20 & 5.88 & 0.04 & & & & & 2.94 & 0.01 & & \\
\hline Cncotopussp2 & & & 4.70 & 0.30 & 20.58 & 0.73 & 5.88 & 0.04 & 20.50 & 0.17 & & & & & & & & & & & & \\
\hline P.barbimanus & 8.82 & 0.08 & 7.64 & 0.40 & 41.17 & 5.62 & & & 26.47 & 0.39 & 47.05 & 2.99 & 35.29 & 1.37 & & & 16.66 & 0.04 & 41.17 & 0.82 & & \\
\hline P.limbatellus & & & & & 5.88 & 0.08 & 32.35 & 0.99 & & & & & & & & & & & 0.04 & & & \\
\hline Chironominisp1 & & & & & & & & & & & & & & & & & & & 2.94 & 0.80 & & \\
\hline C.riparius & 5.88 & 1.11 & 5.29 & 2.70 & 38.23 & 3.27 & 20.58 & 0.55 & 35.29 & 2.67 & 11.76 & 0.27 & 29.41 & 1.38 & 25.00 & 0.32 & & & 2.94 & 0.01 & & \\
\hline P. laetum & & & 6.47 & 2.50 & 47.05 & 1.41 & 44.11 & 2.31 & 38.23 & 0.69 & 38.23 & 7.97 & 29.41 & 0.79 & & & 83.33 & 11.16 & 44.11 & 1.55 & & \\
\hline P. pullum & 4.11 & 3.59 & & & & & & & & & & & & & & & & & & & & \\
\hline C.atridorsum & 2.94 & 8.77 & 0.58 & 1.20 & 14.70 & 0.57 & 23.52 & 1.02 & 32.35 & 1.58 & 23.52 & 0.81 & 8.82 & 0.38 & & & & & 32.35 & 1.24 & & \\
\hline C.mancus & & & & & & & & & & & & & & & & & 75.00 & 16.61 & & & & \\
\hline Tejuncidus & 4.11 & 3.63 & 4.70 & 2.30 & 2.94 & 0.04 & 38.23 & 15.91 & 8.82 & 0.07 & 23.52 & 0.50 & 8.82 & 0.26 & & & & & 32.35 & 1.26 & & \\
\hline Tanytarsus sp2 & & & 3.52 & 4.20 & 35.29 & 7.15 & 32.35 & 1.69 & 52.94 & 52.18 & 23.52 & 1.43 & 47.05 & 2.99 & & & 83.33 & 4.16 & 23.52 & 0.72 & & \\
\hline Dasyhelea sp1 & 9.41 & 1.81 & 3.52 & 1.20 & 2.94 & 0.13 & 17.60 & 2.89 & 17.60 & 2.55 & 5.88 & 2.21 & 11.76 & 1.09 & 16.66 & 0.61 & & & 14.70 & 0.37 & & \\
\hline Dasyheleasp2 & & & & & & & & & & & 2.94 & 0.06 & 11.76 & 0.22 & & & 33.33 & 1.59 & 5.88 & 0.03 & & \\
\hline Ephydra so. & & & & & & & & & & & & & & & & & & & & & 44.11 & 100 \\
\hline
\end{tabular}


anteriores en charcas naturales (0.81-3.35), y ligeramente superiores a los calculados por STRET \& TITMUS (1979) para las comunidades de Qironómidos durante el primer año de la colonización de charcas artificiales.

Los valores de máxima diversidad corresponden a los estanques II y III, los dos únicos medios estudiados con macrófitos. La presencia de vegetación acuática incrementa la heterogeneidad de estos medios, aumentando el rango de microhábitats disponibles para las especies, y limitando diferentes especies a microhábitats particulares, lo cual disminuye la predominancia de las especies (STRET \& TITMUS, 1979).

\section{AGRADECIMIENTOS}

A los distintos especialistas que confirmaron las determinaciones de las especies: Dra. M" Angeles Puig (Ephemeroptera), Dra. Ma Luisa Suárez (Odonata), Sebastián López (Culicidae) y Oscar Soriano (Chironomidae).

\section{BIBLIOGRAFÍA}

BARNES, L.E. 1983. The colonization of ball-clay ponds by macroinvertebrates and macrophytes. Freshwater Biology, 13 : $561-578$.

BEGON, M; HARPER, J.L. \& TOWNSEND, L.C. 1988 Ecología. Individuos, poblaciones y comunidades. Ed. Omega. Barcelona. 886 pp.

BERTRAND, H. 1954. Les insectes aquatiques d'Europe. Encyclopedie Entomologique. Ed. Paul Lechevalier. Paris. Vol. I: 556 pp.; vol II : 547 pp.

BIRD, G.A. \& HYNES, H.B.N. 1981. Movements of adult aquatic insects near streams in southern Ontario. Hydrobiología, 77 : 65-69.

CABALlerO, A. 1919. La Chara faetida A. Br. y las larvas de Stegeomyia, Culex y Anopheles. Bol. Roy. Soc. Esp. Hist. Nat. Madrid 19(8) : 449.

FISHER, S.G. 1983. Sucession in Streams. In: Application and testing of general ecological theory. (J.R. BARNES \& G.W. MINSHALL, eds.): 7-27. Plenun Press. New York.

FISHER, S.G. 1990. Recovery proccesses in lotic ecosystems: Limits of successional theory. Environmental Management, 14(5) : 725-736.

FISHER, S.G.; GRAY, L.J. GRIMN, N.B. \& BUSCH, D.E. 1982. Temporal succession in a desert stream ecosystem following flash fooding. Ecological Monographs, 52 : 93-110.

FRIDAY, L.E. 1987. The diversity of macroinvertebrate and macrophyte communities in ponds. Freshwater Biology, 18 : 87-104.
GRAY, L.J \& FISHER, S.G. 1981. Postflood recolonization pathways of macroinvertebrates in a lowland Sonoran Desert stream. The American Midland Naturalist, 106 (2) : 249-257.

HUTCHINSON, G.E. 1981. Thoughts on aquatic insect. Bioscience, 31(7) : 495-500.

JOHNSON, C.G. 1969. Migration and dispersal of insects by flight. Methuen. London. 763 pp.

MACAN, T.T. 1975. Invertebrados de agua dulce. Eunsa. $118 \mathrm{pp}$.

MALMQVIST, B.; RUNDLE, S.; BRONMARK, C. \& ERLANDSSON, A. 1991. Invertebrate colonization of a new, man-made stream in southern Sweden. Freshwater Biology, 26 : 307-324.

MARGALEF, R. 1977. Ecología. Ed. Omega. Barcelona. $951 \mathrm{pp}$.

McLACHLAN, A.J. \& CANTRELL, M.A. 1980. Survival strategies in tropical rain pools. Oecología, 47 : 344-351.

MILNER, A.M. 1987. Colonization and ecological development of new stream in Glacier Bay Nacional Park, Alaska. Freshwater Biology, 18 : 53-70.

MÜLLER, K. 1982. The colonization cycle of freshwater insects. Oecologia, 52 : 202-207.

ORTEGA, M., SUÁREZ, M.L., VIDAL-ABARCA, M.R. \& RAMÍREZ-DÍAZ, L. 1991. Aspectos dinámicos de la composición y estructura de la comunidad de invertebrados acuáticos de la Rambla del Moro después de una riada (cuenca del río Segura: SE de España. Limnética 7 : 11-25.

PARDO, L. 1923. Observaciones acerca de la acción de la Chara sobre las larvas de los mosquitos. Bol. Soc. Esp. de Hist. Nat. Tomo XXIII.

PATERSON, C.G. \& FERNANDO, C.H. 1969. The macroinvertebrate colonization of a small reservoir in Eastern Canada. Internationale Vereinigung für Theorchische und Angewandte Limnologie Verhandlungen, 17 : 126-136.

PIANKA, E.R. 1970. On r- and k-selection. American Naturalist, 104 : 592-597.

PIELOU, E.C. 1977. Mathematical ecology. J. Wiley \& Sons, N. York, $385 \mathrm{pp}$.

RAMÍREZ-DÍAZ, L. et al. 1992. Los humedales de la región de Murcia. Caja de Ahorros del Mediterráneo. Murcia, $109 \mathrm{pp}$.

ROS, J.D. 1979. Practicas de ecología. Omega. Barcelona.

SHELDON, A. 1984. Colonization dynamics of aquatic insects. In: The ecology of aquatic insects. (REH, V.H. \& ROSENBERG, D.M. eds.): 401-428. Praeger. New York. 625 pp.

STREET, M. \& TITMUS, G. 1979. The colonization of experimental ponds by Chironomidae (Diptera). Aquatic Insects, 1 : 233-244. 
SUÁREZ, M.L. et al. 1991. Diversidad biológica en pequeños cuerpos de agua de regiones áridas y semiáridas: el caso de la Región de Murcia (SE de España). En: Diversidad biológica (F.D. PINEDA, M.A. CASADO, J.M. DE MIGUEL \& J. MONTALVO, eds.) :189-192. Fundación Ramón Areces. Madrid.

THIERY, A. 1978. Etude des communates des invertébrés aquatiques dans differents biotopes des marais $d u$ Plan $d u$ Bourg. These de specalite Ecologie. Univ. de Droit d'Economie et des Sciences, Marseille.

VELASCO, J. 1986. Experiencias sobre colonización por insectos acuáticos de pequeños estanques en el SE. de España (Murcia). Tesis de Licenciatura. Universidad de Murcia. 118 pp.

VELASCO, J. 1989. Estudio de los procesos de colonización de medios artificiales por insectos acuáticos en el Sureste Ibérico. Tesis Doctoral, Universidad de Murcia, 502 pp.
WIGGINS, G.B.; MACKAY, R.J. \& SMITH, J.M. 1980. Evolutionary and ecological strategies of animals in annual temporary pools. Archiv fur Hydrobiolgia, Suppl., 58 (1-2): 87206.

WILLIAMS, D.D. 1977. Movements of benthos during the recolonization of ternporary streams. Oikos, $29: 306-312$.

WILLIAMS, D.D. 1980. Temporal patterns in recolonization of stream benthos. Archiv fur Hydrobiologia, 90 : 56-74.

WILLIAMS, D.D. 1987. The ecology of temporary waters. Timber Press, Portland, 205 pp.

WILLIAMS, D.D. \& HYNES, H.B.N. 1976. The recolonization mechanisms of stream benthos. Oikos, 27 : 265-272.

WILLIAMS, W.D. 1985. Biotic adaptations in temporary lentic waters, whith special reference to those in semiarid regions. Hydrobiologiu, $125: 85-110$. 